\title{
Der Kasuswert des prädikativen Adjektivs in Infinitivkonstruktionen des Polnischen
}

\author{
The case of the predicative adjective \\ in Polish infinitive constructions
}

\begin{abstract}
The following paper addresses the issue of constructions with predicative adjectives in Polish. The main focus of attention will be put on adjectives which are governed by the copula in the infinitive form, as here one can observe some interesting casus alternation between the nominative and the instrumental. On the basis of the author's own empirical analysis it will be examined which factors determine the choice of the casus. As it seems it is both the syntactic status of the infinitive construction and lexical factors.
\end{abstract}

KEYWORDS: predicative adjective, case, secondary predication, control.

\section{EINLEITUNG}

Adjektiv (Beiwort, Eigenschaftswort, Artwort, Wiewort) ist eine grammatische Kategorie, die Wörter umfasst, die syntaktisch beim Nomen stehen (attributive Verwendung (i) der kleine Hund, mały pies) oder von einer Kopula regiert werden (prädikative Verwendung (ii) Der Hund ist klein. Pies jest mały). Ansonsten können sie adverbial ((iii) Die Kinder singen schrecklich. Dzieci strasznie śpiewają) verwendet werden. Syntaktisch gesehen fasst also die Klasse des Adjektivs mindestens drei ihren Grundfunktionen, d.h. der attributiven, prädikativen und adverbialen, entsprechende Subklassen zusammen.

Während in der deutschen Grammatikschreibung ein Konsens darüber zu herrschen scheint, dass adverbial gebrauchte Wörter wie schrecklich in (iii) 
Adjektiven zugeordnet werden ${ }^{1}$, gestaltet sich die Behandlung von Wörtern wie strasznie (schrecklich) in (iii) in den Grammatiken des Polnischen nicht einheitlich. Durch die Suffigierung der ,Adjektive' in adverbialer Funktion werden sie mitunter zur Klasse der Adverbien gerechnet (vgl. Grzegorczykowa 1975; Laskowski 1999: 64) oder zu adverbialen Formen der adjektivischen Lexeme (poln. formy przysłówkowe leksemów przymiotnikowych, Saloni, Świdziński 1998: 105). In der konfrontativen Monografie von Bzdęga (1980) werden sie zu Vertretern des Adjektivparadigmas in adverbialer Funktion gezählt, wohl mit der Absicht, eine homogene Beschreibungsebene für die konfrontierten Sprachen zu schaffen. Auch in diesem Beitrag werden adverbial gebrauchte Adjektive den "reinen“ Adjektiven zugeordnet. In straszni-e bringt beispielsweise das Suffix $-e$ die adverbiale Funktion zum Ausdruck im Unterschied zum attributiven und prädikativen straszn-y, bei dem $-y$ kein Suffix, sondern die Flexionsendung Nom.Mask.Sg.Pos. ist.

\section{GEGENSTANDSBESTIMMUNG}

In diesem Beitrag wird diskutiert, welchen Kasuswert die von einer Kopula regierten Adjektive annehmen können und ob sich diesbezüglich gewisse Regularitäten feststellen lassen. Das Hauptaugenmerk wird dabei auf polnische Daten und Grammatikalitätsurteile gerichtet, weil im Deutschen prädikative Adjektive, sei es in finiten oder in infiniten Kopulasätzen, stets in der unmarkierten Grundform erscheinen. Dies trifft auf das Polnische nicht zu. Vgl.:

(1) Peter ist fleißig.

(1)a. Es ist von Vorteil, fleißig zu sein.

(2) Piotr jest piln-y.

,Peter ist fleißig (Nom)'

(2)a. Jest korzystnie być piln-ym.

'ist gut sein fleißig (Instr.)'

In (2), d.h. in einem finiten Satz, steht das Adjektiv in der Nominativform. Ist es aber von der Kopula im Infinitiv abhängig, erscheint es im Instrumental.

${ }^{1}$ Dies mag u.a. mit fehlender morphologischer Markierung von adverbial gebrauchten Adjektiven zusammenhängen. Heute sind nur lange und sachte in dieser Funktion suffigiert (vgl. Bzdęga 1980: 7). 


\section{FORSCHUNGSÜBERBLICK UND EIGENE UNTERSUCHUNG}

Alternationen wie die in (2) und (2a.) waren bereits Gegenstand linguistischer Forschungen, bis jetzt wurde aber keine einheitliche Erklärung für dieses Phänomen geboten. In Saloni, Świdziński (1998) wird z.B. lediglich konstatiert, dass der Infinitiv być regierten Adjektiven zwei alternative Kasus, d.i. den Nominativ oder Instrumental, zuweisen kann. Dennoch können die beiden Kasus mitnichten frei ausgetauscht werden und unterliegen starken kontextuellen Bedingungen. Welche Bedingungen es sind, wird nicht erörtert. Allerdings beschränkt sich diese Kasusalternation nicht nur auf von być regierte Adjektive, sondern sie ist auch bei anderen (kopulaähnlichen) Verben festzustellen, z.B. bei czuć się (sich fühlen):

(3) Pragnął czuć się szczęśliwy / szczęśliwym.

$(\mathrm{er})^{2}$ wollte fühlen sich glücklich Nom / Instr

Aus deskriptiver Sicht beschäftigt sich mit solchen Erscheinungen u.a. Gębka-Wolak (2003). Sie bietet v.a. eine Distributionsbeschreibung der alternierenden Kasusformen und versucht den Ursachen einer solchen Verteilung auf den Grund zu gehen. Adjektive im Instrumental seien sprachhistorisch älter und die heutige Alternation sei eine Folge der Ersetzung des Instrumentals durch den Nominativ (vgl. a.a.O.: 111, Anm. 4). Synchron gesehen seien für eine solche Verteilung u.a. stilistische Faktoren verantwortlich, d.i. der Instrumental werde im gehobenen, poetischen Stil vorgezogen und dies unabhängig davon, ob das Adjektiv vom finiten oder infiniten Verb regiert wird:

(4) Lecz wielka dzieli nas granica, boś ty bogatą przecież jest. ${ }^{3}$

'aber große trennt uns Grenze, weil du reich Instr doch bist'

(5) [...] znalazła się brzemienną za sprawą Ducha Świętego (Matthäus 1, 18)

'(Maria) wurde schwanger Instr von dem heiligen Geist'

Ansonsten seien grammatische und informationsstrukturelle Faktoren im Spiel. Zu den grammatischen gehört der Kasus der NP, die mit dem Adjektiv in Genus und Numerus kongruiert (a.a.O.: 116):

\footnotetext{
${ }^{2}$ Im Polnischen als einer pro-drop-Sprache wird das aus dem Kontext identifizierbare Subjekt meist ausgelassen.

${ }^{3}$ Aus einem populären Lied.
} 
(6) [Nowa studentka] $]_{\text {Nom }}$ mogła wreszcie zostać doceniona / docenioną przez profesora.

'Neue Studentin konnte endlich werden geschätzt Nom / Instr durch Professor'

Hier liegt entweder ein "Kasustransfer" vor, d.h. der Nominativ wird von der NP an das Adjektiv weitergegeben oder es steht alternativ im Instrumental. Zu den Verben, die bei einer nominativischen Subjekt-NP Adjektive im Nominativ oder Instrumental regieren, gehören nach GębkaWolak (2003: 112) folgende Kopula- und kopulaähnliche Verben: by(wa)ć (sein), (po)czuć się (sich fühlen), okazać się (sich herausstellen), pozostać (bleiben), sta(wa)ć się (werden), wyda(wa)ć się / zda(wa)ć się (scheinen).

Steht die übergeordnete NP in einem anderen Kasus als Nominativ, so bekommt das Adjektiv ausschließlich den Instrumental zugewiesen:

(7) Tomasz nie pozwalał Pawłowi Dat być modnym Instr / *modny Nom.

'Tomasz nicht erlaubte Paweł sein modisch Instr / "Nom'

(8) Czesiek nauczył mnie Akk być asertywnym Instr / *asertywny Nom.

'Czesiek brachte mir bei sein selbstbewusst Instr / *Nom'

Von den informationsstrukturellen Faktoren könnte die Fokussierung des Themas genannt werden, die ebenfalls den Instrumental nach sich zieht bzw. nahe legt, denn der Nominativ ist nicht ungrammatisch, sondern markiert:

(9) Na stadionie Legii rozegrano mecz między drużynami piłkarskimi aktorów i dziennikarzy.

'Im Stadion von Legia wurde ein Fußballspiel gespielt zwischen Mannschaften der Schauspieler und Journalisten'

(9)a. Lepszymi FokInstr / ?Lepsi FokNom okazali się aktorzy.

‘Besser FokInstr / ?besser FokNom zeigten sich Schauspieler' (Gębka-Wolak 2003: 114).

Die Beispiele veranschaulichen, dass bezüglich der Kasuswahl beim prädikativen Adjektiv im Polnischen eine beachtenswerte Dynamik im Spiel ist, d.h. verschiedene Faktoren üben Einfluss auf seine Form aus.

Bondaruk (2004) beschränkt sich in ihrer generativ ausgerichteten Arbeit nicht nur auf die Deskription, sondern sie bemüht sich auch unter Rekurs auf theorieinterne Kategorien, die in Frage stehende Kasusaltertanz zu erklären. Das Vorkommen des Instrumentals macht sie u.a. von der Präsenz des Komplementierers żeby (S. 200) abhängig, und zwar so, dass dieser Kasus in żeby-Infinitivkonstruktionen mit dem Vorkommen eines lexikalisch unausgedrückten PRO-Subjekts korreliert: 
(10) Stefan błagał, $\mathrm{PRO}_{\mathrm{arb}}{ }^{4}$ żeby być rozsądnym / * rozsądny.

'Stefan flehte $\mathrm{PRO}_{\mathrm{arb}}$, dass sein vernünftig Instr / *Nom'

Aber auch ohne żeby kann der Instrumental erscheinen:

(11) Dobrze (jest) $\mathrm{PRO}_{\text {arb }}$ być pięknym / *piękny.

'Gut (ist) $\mathrm{PRO}_{\text {arb }}$ sein schön Instr / *Nom'

Auch wenn die Kopula als Gerundium realisiert wird, erscheint das abhängige Adjektiv im Instrumental:

(12) $\mathrm{PRO}_{\text {arb }}$ Bycie pięknym / *piękny to miła rzecz.

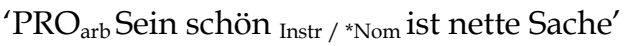

Aus den Gegebenheiten in (10)-(12) zieht Bondaruk den Schluss, dass der identische Kasus in den drei Konstruktionen ein Indiz dafür ist, dass die Teilsätze über das gleiche Subjekt verfügen, nämlich $\mathrm{PRO}_{a r b}$. Es soll für den Instrumental verantwortlich sein.

Im Unterschied dazu weist das lexikalisch unausgedrückte Subjekt in finiten Sätzen (= pro) den Nominativ zu (vgl. Franks 1995: 276; Bondaruk 2004: 201):

(13) pro Jest piękny / rozsądny / *pięknym / *rozsądnym.

'(er) pro Ist schön Nom / *Instr / vernünftig Nom / *Instr'

(14) pro Marzyłem, żeby mój syn był mądry / *mądrym.

'(ich) pro Träumte, dass mein Sohn ist klug Nom / *Instr'

Auch PRO kann mit dem Nominativ kookkurrieren, allerdings wenn es in Infinitivkonstruktionen bei Subjektkontrollverben ${ }^{5}$ vorkommt (Bondaruk 2004: 229-233):

(15) Marek chciał PRO być mądry / *mądrym.

'Marek wollte PRO sein klug Nom/*Instr'

Die von mir ausgewerteten Umfragen (s.u.) deuten allerdings darauf hin, dass zumindest manche Behauptungen von Bondaruk relativiert werden sollen, z.B. verlangt PRO entgegen Bondaruks Annahmen bei obiecać (ver-

${ }^{4} \mathrm{PRO}_{\text {arb }}$ (arbiträres PRO) bedeutet, dass die Referenz des leeren Infinitivsubjekts nicht durch innersprachliche strukturelle Faktoren, sondern durch den außersprachlichen Kontext festgelegt wird.

${ }^{5}$ Es sind Verben, deren Subjektreferenz mit der des eingebetteten Infinitivsubjekts PRO zusammenfällt. 
sprechen) als einem klassischen Subjektkontrollverb vornehmlich das Adjektiv im Instrumental:

(16) Marek obiecał matce być ?uważny / uważnym.

'Marek versprach der Mutter sein aufmerksam ?Nom / Instr'

Infinitivkonstruktionen als Komplemente der Objektkontrollverben ${ }^{6}$ enthalten prädikative Adjektive im Instrumental:

(17) Maria kazała Markowi być mądrym / *mądry.

'Maria ließ Marek sein klug Instr / 'Nom'

Auch Franks (1995) befasst sich in seiner Arbeit zur slawischen Morphosyntax mit dem Kasuswert des prädikativen Adjektivs. Im Großen und Ganzen stimmen seine Einsichten mit denen anderer Autoren überein, soweit er die entsprechenden Phänomene anhand korrekter Beispiele erklärt. Bedauerlicherweise versucht er auch aufgrund von ungrammatischen Beispielen auf bestimmte Regelmäßigkeiten der polnischen Grammatik zu schließen (vgl. Franks 1995: 277):

(18) Poprosiłem Jana PRO być *szczery / szczerym / *szczerego.

'(Ich) bat Jan PRO sein ehrlich *Nom / Instr / *Akk'

Im Polnischen lässt das Verb (po)prosić (bitten) im Gegensatz zum Deutschen keinen Infinitivsatz als Objekt zu (vgl. Morciniec et al. 1995: 83), daher können offenbar aus dem o.g. Beispiel keinerlei Schlussfolgerungen gezogen werden. Nach Zaron (1980: 42f.) kann (po)prosić zwar mit Infinitiv vorkommen, jedoch lediglich, wenn die Infinitivkonstruktion durch żeby eingeleitet wird:

(19) Ania poprosiła Waldka (o to), żeby pomóc jej.

'Ana bat Waldek (darum), dass helfen ihr'

Dieser Satz klingt aber etwas ungewöhnlich, der finite Nebensatz scheint gelungener zu sein:

(20) Ania poprosiła Waldka (o to), żeby pomógł jej.

'Ana bat Waldek (darum), dass (er) hilft ihr'

Was Nominalisierungen der den Infinitiv regierenden Verben anbetrifft, so hat man es mit zahlreichen Idiosynkrasien zu tun, d.h. die Infinitivrektion wird nicht immer an das Nomen vererbt (vgl. auch Gębka-Wolak 2011: 57):

${ }^{6}$ D.h. Verben, die im unmarkierten Fall eine Referenzidentität zwischen dem Objekt des übergeordneten Verbs und dem Subjekt des Infinitivs aufweisen. 
(21) Jan obiecał PRO być ?szczery / szczerym. $\rightarrow$ *Jan złożył obietnicę PRO być *szczery / szczerym.

'Jan versprach PRO sein ehrlich ?Nom / Instr $\rightarrow$ *Jan machte Versprechen PRO sein ehrlich *Nom / Instr'

Franks (1995: 279) scheint aber dies verkannt zu haben, denn er stuft ein Beispiel wie (21), bei ihm 126a, als grammatisch ein. Mehr noch, unter Berufung auf Comries "cohesion principle“ führt er aus, dass für die Ungrammatikalität der Nominativform des Adjektivs die entfernte Stellung der mit PRO koreferenten NP Jan verantwortlich sei. Die größere Distanz zwischen dieser NP und PRO soll den Transfer des Nominativs erschweren, woraus der ungrammatische Nominativ und der grammatische Instrumental resultieren. Dies ist selbstverständlich nicht korrekt, denn der ganze Satz ist ungrammatisch, nicht nur mit dem Nominativ.

Dennoch sind im Polnischen Infinitivkomplemente von Nomina generell möglich, z.B. nach możliwość (Möglichkeit):

(22) Spróbuj uporządkować dzienny rytm psa, tak aby miał możliwość PRO odpocząć także sam.

'Versuche ordnen den Tagesrhythmus deines Hundes so, dass (er) hat Möglichkeit PRO sich erholen auch selbst'

Aus der präsentierten Übersicht dürfte hervorgegangen sein, dass im Bereich des Kasuswerts der prädikativen Adjektive keine Einigkeit besteht, und dies sowohl im Sprachgebrauch als auch in der Behandlung des Themas durch Sprachwissenschaftler. Um dem teilweise abzuhelfen, wurde für die Zwecke dieses Aufsatzes eine Umfrage durchgeführt, die aus 30 Sätzen mit prädikativen Adjektiven besteht. Die Adjektive erscheinen in verschiedenen strukturellen Konfigurationen, d.h. sie sind Ergänzungen sowohl von finiten Verben als auch von Infinitiven in Kontrollkonstruktionen. In einem Satz erscheint das Adjektiv als Komplement des Gerundivs bycie (Sein). Die 106 Befragten sollten sich jeweils für eine Kasusvariante entscheiden und das Wort unterstreichen, das sie am wahrscheinlichsten auch selbst verwenden würden. Z.B. im Satz Marek nakazał Zosi być uprzejma / uprzejma (Marek ließ Zosia sein höflich Instr/Nom) sollten die Probanden eins der fettgedruckten Wörter unterstreichen.

Die Erhebung sollte als stichprobenartig angesehen werden, die u.U. auf gewisse Präferenzen schließen lässt, sie erhebt daher keinen Anspruch auf Repräsentativität. Sämtliche Schlussfolgerungen beziehen sich demzufolge allein auf die Stichprobe.

Die Auswertung der Umfrage hat in Bezug auf bestimmte Konstruktionen mitunter zu überraschenden Ergebnissen geführt. So erscheint bei- 
spielsweise in Sätzen mit Subjektkontrollverben chcieć (wollen), lubić (mögen), marzyć (träumen), mieć zamiar (die Absicht haben), obiecać (versprechen), pragną́ (begehren), starać się (sich bemühen) das von dem eingebetteten Infinitiv regierte Adjektiv nicht immer im Nominativ wie von Bondaruk (s.o.) angenommen. Die Kasusverteilung scheint hier vielmehr eine lexikalische Angelegenheit zu sein. Dies möge die folgende Tabelle veranschaulichen:

\begin{tabular}{|l|c|c|c|c|c|c|c|}
\hline \multicolumn{1}{|c|}{ Verb } & chcieć & lubić & marzyć & $\begin{array}{c}\text { mieć } \\
\text { zamiar }\end{array}$ & obiecać & pragnąć & starać się \\
\hline Nom. & $95 \%$ & $88 \%$ & $48 \%$ & $77 \%$ & $29 \%$ & $88 \%$ & $81 \%$ \\
\hline Instr. & $5 \%$ & $12 \%$ & $52 \%$ & $23 \%$ & $71 \%$ & $12 \%$ & $19 \%$ \\
\hline
\end{tabular}

Was besonders auffällt, ist die große Diskrepanz im Kasus des infinitivregierten Adjektivs in Abhängigkeit vom übergeordneten Kontrollverb. Die zwei Extreme sind Verben chcieć und obiecać. Im Satz

(23) Jurek chce być przystojny / przystojnym.

,Jurek will sein guttaussehend Nom/Inst' $^{\prime}$

haben sich 95\% der Befragten für die nominativische Variante entschieden, obwohl der Instrumental nicht ungrammatisch ist, sondern stilistisch gehoben. Dennoch haben lediglich 5\% Prozent der Probanden den Instrumental gewählt. Bei obiecać (Marek obiecat matce być rozsądny / rozsądnym. dt. ,Marek versprach der Mutter sein vernünftig Nom / Instr') dagegen haben 71\% Personen das Adjektiv im Instrumental als die bevorzugte Variante unterstrichen. Bei anderen Verben sind die Unterschiede nicht so auffällig, dennoch kann man schlussfolgern, dass Grammatikalisierungsprozesse hinsichtlich des prädikativen Adjektivs nach Subjektkontrollverben im Polnischen noch nicht abgeschlossen sind.

Bei Objektkontrollverben scheint die Situation nicht so facettenreich zu sein, denn beispielsweise bei nakazać (befehlen) haben alle Personen bis auf eine auf den Instrumental als den Kasus des prädikativen Adjektivs beim Infinitiv hingewiesen. Dies entspricht auch den Einsichten, die in der Literatur präsentiert werden (vgl. u.a. Bonadruk 2004: 201).

Unterschiedlich werden die Kasusverhältnisse bewertet, wenn das Adjektiv von der Kopula być (sein) in Konstruktionen mit frei interpretierbarem PRO regiert wird, z.B. haben die Befragten im Satz

(24) Dobrze jest być *młody / młodym.

,gut ist sein jung *Nom / Instr' 
zu 100\% den Instrumental gewählt, d.h. in Infinitivkonstruktionen mit arbiträrem PRO, die in Subjektfunktion auftreten, scheinen im Lichte der Umfrage die Grammatikalisierungsprozesse bezüglich des prädikativen Kasus bereits abgeschlossen zu sein.

Identische Grammatikalitätsurteile liegen vor, wenn das Subjekt durch das Gerundium bycie (Sein) konstruiert wird:

(25) Bycie *przygotowany / przygotowanym to mój obowiązek.

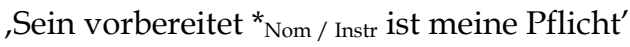

In (25) haben alle Probanden ebenfalls den Instrumental als den bevorzugten Kasus gekennzeichnet.

Ähnliche grammatische Präferenzen liegen vor, wenn die mit PRO koreferente Phrase in Form einer Präpositionalphrase auftritt wie in

(26) Dla mnie 1 jest ważne $\mathrm{PRO}_{1}$ być ?szczęśliwy / szczęśliwym.

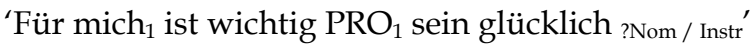

In (26) haben nämlich 102 von 106 Probanden ebenfalls den Instrumental gewählt.

Aufgrund von (24)-(26) kann geschlussfolgert werden, dass der Instrumental als unmarkierter Kasus des prädikativen Adjektivs gewählt wird, wenn es von der Kopula in Infinitiv- oder Gerundivform abhängig ist.

Fungiert die Infinitivkonstruktion als Objekt oder Adverbialsatz, hat man es mit einer unvergleichbar größeren Varianz zu tun, denn z.B. in

(27) Hania nie wie, czy PRO być szczera / szczerą.

'Hania nicht weiß, ob PRO sein ehrlich Nom / Instr'

haben sich 58\% für den Instrumental entschieden. Dieser Kasus überwiegt ebenfalls, wenn der Infinitiv als Adjunkt fungiert, wie in

(28) Ciężko pracuje, żeby PRO być bogaty / bogatym.

'(er) arbeitet schwer, um PRO sein reich Nom / Instr'

Hier haben 71\% den Instrumental gewählt.

Die Wahl des einen oder anderen Kasus scheint nicht unbedingt von der Präsenz des Komplementierers żeby abhängig zu sein, wie von Bondaruk (2004: 200) angenommen wird, denn im Satz

(29) Marek marzy, żeby być najlepszy / najlepszym z gramatyki.

'Marek träumt, dass sein der beste Nom / Instr in Grammatik'

verteilen sich die beiden Kasus fast gleichmäßig (s. Tabelle oben), d.h. żeby verursacht nicht immer die Bevorzugung des Instrumentals. 
Ein interessanter Kontrast wurde in zwei strukturell identischen Konstruktionen festgestellt, in denen sich die Subjekte lediglich im grammatischen Genus unterscheiden:

(30) Chłopiec jest za mały, żeby być rozsądny / rozsądnym.

'Junge ist zu klein, um sein vernünftig Nom / Instr'

(31) Dziecko jest za małe, żeby być rozsądne / rozsądnym.

,Kind ist zu klein, um sein vernünftig Nom / Instr'

Im Falle von (30) haben 70\% den Instrumental vorgezogen, bei (31) dagegen nur haben lediglich 15\% diesen Kasus gewählt.

\section{AUSBLICK}

Aus den obigen Ausführungen geht hervor, dass im Bereich des morphologischen Kasus des prädikativen Adjektivs im Polnischen Grammatikalisierungsprozesse im Gange sind, deren Ende noch nicht abzusehen ist. Lediglich im Falle der arbiträren Kontrolle scheinen sich die Polnischsprecher auf den Instrumental festgelegt zu haben. In anderen Konstruktionen ist die Kasuswahl von solchen Faktoren wie der Kontrolltyp, die thematische Struktur der Äußerung oder das grammatische Genus des durch das Adjektiv modifizierten Nomens zu sein.

Da mit dem steigenden Prestige und Wirtschaftsaufkommen Polens eine größere Nachfrage nach Polnisch als Fremdsprache Hand in Hand geht, könnten die Ergebnisse der vorliegenden Analyse nach didaktischer Aufarbeitung in Polnischlehrewerken für Fortgeschrittene berücksichtigt werden.

\section{LITERATURVERZEICHNIS}

Bondaruk, A., 2004. PRO and Control in English, Irish and Polish - A Minimalist Analysis. Lublin: Wydawnictwo KUL.

Bzdęga, A.Z., 1980. Das Adjektiv im Polnischen und Deutschen. Versuch einer Konfrontation. Wrocław-Warszawa-Kraków-Gdańsk: Ossolineum.

Franks, S., 1995. Parameters of Slavic Morphosyntax. New York-Oxford: Oxford University Press.

Gębka-Wolak, M., 2003. Zakres użycia niemianownikowej formy przymiotnika w pozycjach konotowanych przez czasownik. In: Gębka-Wolak, M., Kaproń-Charzyńska, I., Urban, M. (Hrsg.). Studia z gramatyki i leksykologii języka polskiego. Prace dedykowane Profesor Marii Szupryczyńskiej. Torun: Wydawnictwo UMK, 109-122.

Gębka-Wolak, M., 2011. Pozycje składniowe frazy bezokolicznikowej we wspótczesnym zdaniu polskim. Torun: Wydawnictwo Naukowe UMK. 
Grzegorczykowa, R., 1975. Funkcje semantyczne i składniowe polskich przysłówków. WrocławWarszawa-Kraków-Gdańsk: Ossolineum.

Laskowski, R., 1999. Zagadnienia ogólne morfologii. In: Grzegorczykowa, R., Laskowski, R., Wróbel, H. (Hrsg.). Gramatyka wspótczesnego języka polskiego. Morfologia. Warszawa: PWN, 27-86.

Morciniec, N., Cirko, L., Ziobro, R., 1995. Stownik walencyjny czasowników niemieckich i polskich. Wörterbuch zur Valenz deutscher und polnischer Verben. Wrocław: Wydawnictwo UWr.

Saloni, Z., Świdziński, M., 1998. Składnia wspótczesnego jezzyka polskiego. Warszawa: PWN.

Zaron, Z., 1980. Ze studiów nad składnia i semantyką czasownika. Polskie czasowniki z uzupetnieniem werbalnym oznaczające relacje osobowa $z$ argumentem zdarzeniowym. Wrocław-WarszawaKraków-Gdańsk: Ossolineum. 